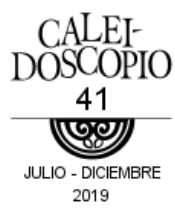

\title{
La resiliencia en adultos con diabetes tipo 2: revisión teórica (2007-2018)
}

Resilience in adults with type 2 diabetes: theoretical review (2007-2018)

ANA MARÍA SANDOVAL JARAMILLO Universidad de Guanajuato, México

RESUMEN

La presente revisión teórica, que abarca entre los años 2007 y 2018, tiene por objetivo conocer los aportes científicos que la resiliencia tiene en su relación con personas adultas que viven con diabetes tipo 2. Se trata de un estudio descriptivo de revisión de artículos para el cual se realizó una búsqueda en las bases de datos: EBSCOhost, ProQuest, Portal Regional BVS (Biblioteca Virtual en Salud) y GALE a través del portal de CONRICyT. En total se identificaron 30 artículos que cumplen los criterios de inclusión establecidos. Se concluye que aún son escasos los estudios que investigan la resiliencia en su relación con la diabetes tipo 2; resalta la productividad de artículos en idioma inglés y de origen estadounidense; la resiliencia se asocia positivamente con el control glicémico; el instrumento más utilizado para medir la resiliencia es la Escala de Resiliencia de Connor-Davidson (CD-RISC), aunque muchos estudios no miden la variable 
directamente. Se considera primordial trabajar en aumentar la resiliencia en pacientes con diabetes tipo 2.

Palabras clave: resiliencia; diabetes tipo 2; adultos; revisión teórica.

\section{ABSTRACT}

The objective of this theoretical review covering the years between 2007 and 2018 is to know about the scientific contributions that resilience has in its relationship with adults living with type 2 diabetes. This is a descriptive study of review of articles for wich a search was made in the databases: EBSCOhost, ProQuest, Portal Regional BVS (Biblioteca Virtual en Salud) y GALE through the CONRICyT portal. In total, 30 articles were identifiied that meet the established inclusion criteria. It is concluded that there are still few studies investigating resilience in relation to type 2 diabetes; highlights the productivity of articles in English and American origin; resilience is positively associated with glycemic control; the most widely used instrument to measure resilience is the Connor-Davidson Resilience Scale (CD-RISC), although many studies do not measure the variable directly. It is considered essential to work on increasing resilience in patients with type 2 diabetes.

Keywords: resilience; type 2 diabetes; adults; theoretical review.

Actualmente, la diabetes tipo 2 es un padecimiento que va en aumento a nivel mundial. Según la Organización Mundial de la Salud (OMS, 2017), el número de personas con diabetes ha aumentado de 108 millones en 1980 a 422 
millones en 2014, pero lo que resulta realmente alarmante es el efecto de la diabetes no controlada, conocida como hiperglucemia, ya que con el tiempo daña gravemente muchos órganos y sistemas. Por lo tanto, se plantea que si no se realizan intervenciones efectivas para el control de este padecimiento, los costos a nivel salud seguirán en aumento $\mathrm{y}$, antes de sobrevenir la muerte en estos pacientes, se darán múltiples complicaciones no sólo físicas, sino también las que generan un impacto emocional, social y económico para el individuo y quienes le rodean.

Por otro lado, la resiliencia es "un proceso de afrontamiento ante la adversidad, cambio u oportunidad que resulta en la identificación, fortificación y enriquecimiento de las cualidades resilientes o de los factores protectores" (Richardson, 2002, p. 308). En el adulto puede ser simultáneamente un proceso, un resultado y un recurso, dependiendo del nivel de análisis y la etapa de su desarrollo Aldwin y Igarashi, 2012).

Desde la investigación basada en la evidencia, se reconoce que la resiliencia es una variable que mejora la salud física y mental en diversos procesos de enfermedad, incluyendo la diabetes tipo 2 (Brix et al., 2008; Jamison, Weidner, Romero y Amundsen, 2007; Kralik, Van Loon y Visentin, 2006).

La importancia de intervenir desde la perspectiva de la resiliencia en pacientes con diabetes radica en que la atención no sólo se centre en el tratamiento de los síntomas, sino en abordar de manera integral a la persona, reconociendo las dimensiones en las que puede desplegar sus potencialidades para ser capaz de responder de manera activa y autogestionaria a los desafíos de la vida diaria (García-Vesga y Domínguez-De la Ossa, 2013), y con 
ello tener un mejor afrontamiento de su padecimiento que le permita estar en control y tener mayor calidad de vida.

Además, la resiliencia como indicador de la habilidad de las personas para soportar las experiencias de gran adversidad, ha llevado al desarrollo e implementación de métodos para mejorarla. Existen diversos recursos y técnicas que se pueden trabajar para promover la resiliencia y reducir el riesgo de diabetes tipo 2 (Steptoe, 2016).

Por lo tanto, esta revisión tiene por objetivo analizar la variable resiliencia en su relación con personas que viven con diabetes tipo 2, al identificar cómo ha sido medida y atendida para ser potenciada a través de intervenciones y de estudios empíricos.

\section{MÉTODO}

\section{Materiales}

Se consultaron las bases electrónicas de datos EBSCOhost, ProQuest, Portal Regional de la Biblioteca Virtual en Salud (BVS), y GALE a través del Consorcio Nacional de Recursos de Información Científica y Tecnológica (CONRICyT) para localizar los artículos de investigación sobre resiliencia y diabetes tipo 2, publicados en revistas científicas.

\section{Diseño}

Se trata de un estudio descriptivo, de revisión de artículos sobre las variables resiliencia y diabetes tipo 2 en adultos, publicados entre los años 2007 y 2018. 
Estrategia de búsqueda

Se buscó homogeneizar la selección de acuerdo con lo permitido por cada base de datos; se resumen los criterios de inclusión en la Tabla 1.

Tabla 1. Criterios de inclusión utilizados en las bases de datos (septiembre-octubre 2018)

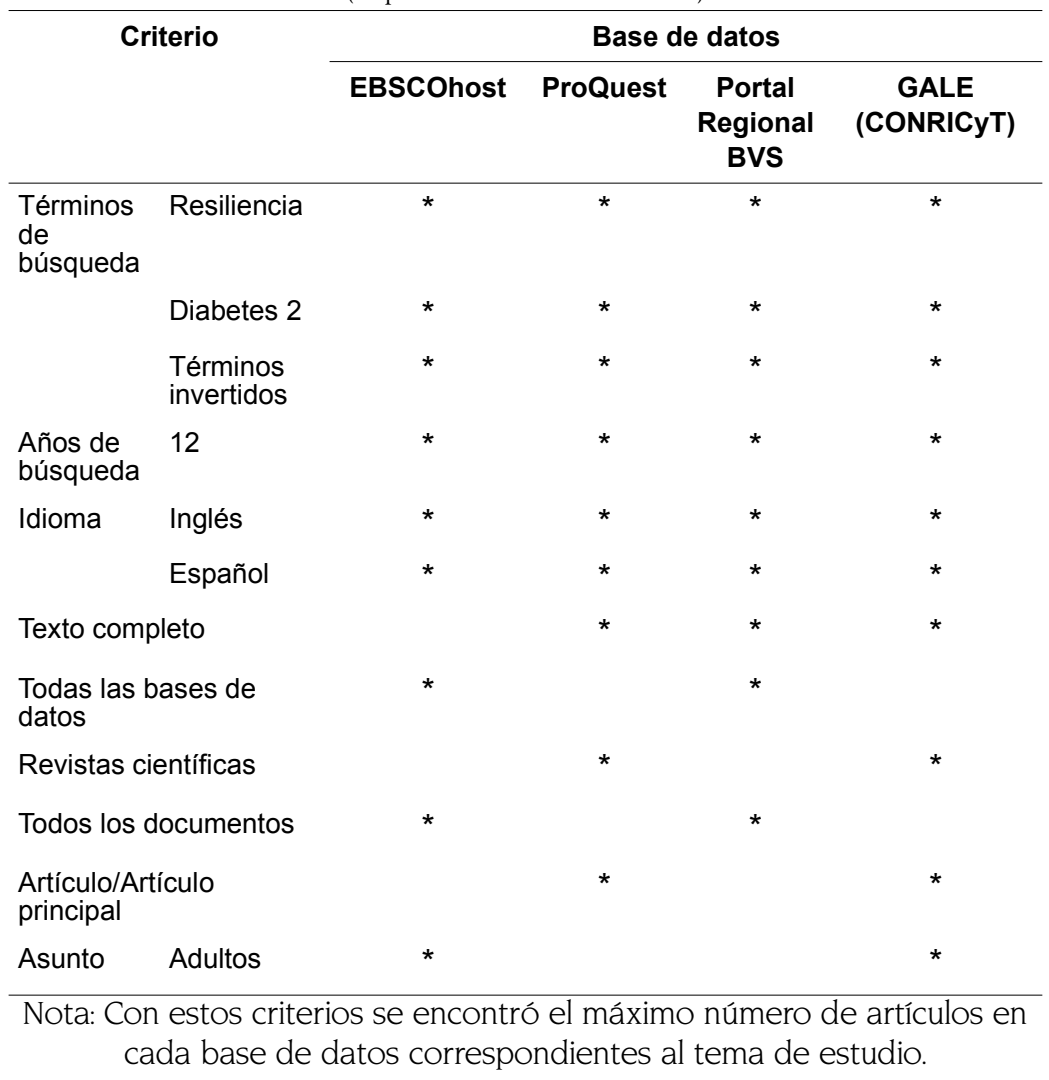

En la Figura 1 se muestra el diagrama de flujo de la selección de artículos incluidos en la presente revisión. 


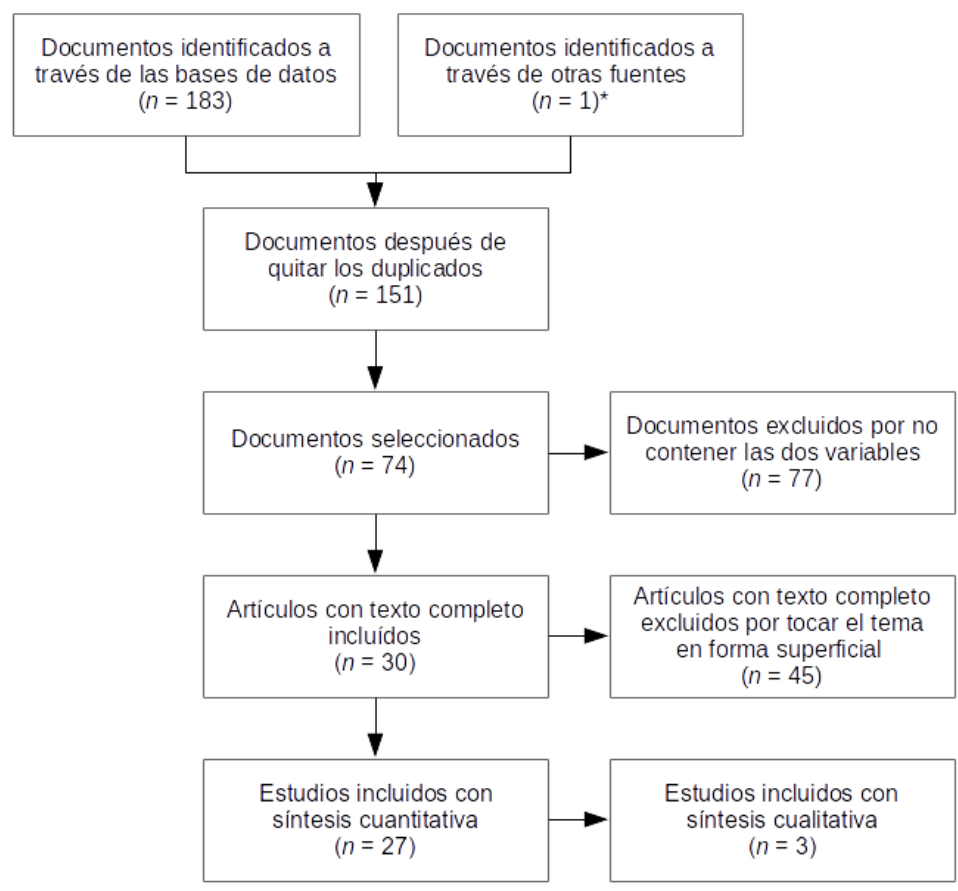

Figura 1. Diagrama de flujo para la selección de estudios sobre resiliencia y diabetes tipo 2 (revisión septiembre-octubre 2018)

*Artículo encontrado en google académico.

\section{RESULTADOS}

En total, se localizaron 183 artículos (Figura 1), de los cuales se identificaron 32 repetidos que fueron descartados, posteriormente se excluyeron los que sólo se relacionaron con alguna de las variables (resiliencia o diabetes 2), después, los que abordaban el tema de estudio de una forma muy superficial por centrarse más en una sola variable, finalmente, sólo quedaron 29 artículos de esta búsqueda y se agregó uno que se localizó de una fuente 
secundaria, dejando para nuestro análisis un total de 30 estudios.

En la Tabla 2 se observa que la mayoría de los estudios de la presente revisión son de tipo cuantitativo-descriptivo ( $n=15 ; 50 \%$ ), le siguen las intervenciones clínicas ( $n=8 ; 26.6 \%$ ), luego los que pertenecen a modelos de trabajo de la resiliencia y a revisiones teóricas ( $n=4 ; 13.3 \%$ ), y finalmente los de tipo cualitativo-exploratorio $(n=3 ; 10 \%)$.

Predominantemente los estudios de tipo cuantitativodescriptivo, de intervención y de modelos para trabajar la resiliencia fueron llevados a cabo en Estados Unidos $(n=13$; 43.3\%), representando un alto porcentaje en comparación con los estudios reportados en nuestra región de América Latina ( $n=2 ; 6.6 \%$ ), los cuales sólo son de tipo cuantitativodescriptivo. La mayoría de las publicaciones están en idioma inglés ( $n=29$; 96.6\%).

Tabla 2. Tipo de estudios y países de los artículos publicados entre 2007 y 2018 sobre resiliencia y diabetes tipo 2

\begin{tabular}{|c|c|c|}
\hline Tipo de estudio & Países & Total \\
\hline $\begin{array}{l}\text { Estudios cuantitativos } \\
\text { descriptivos }\end{array}$ & $\begin{array}{l}\text { Estados Unidos (5), Alemania (1), } \\
\text { Australia (1), Brasil (1), España (1), } \\
\text { Holanda (1), India (1), Irán (1), Israel (1), } \\
\text { Pakistán (1), Venezuela (1). }\end{array}$ & 15 \\
\hline $\begin{array}{l}\text { Estudios sobre intervenciones } \\
\text { clínicas }\end{array}$ & $\begin{array}{l}\text { Estados Unidos (6), Australia (1), Irán } \\
\text { (1). }\end{array}$ & 8 \\
\hline $\begin{array}{l}\text { Modelos de resiliencia y } \\
\text { revisiones teóricas }\end{array}$ & $\begin{array}{l}\text { Estados Unidos (2), Alemania-Grecia } \\
\text { (1), Suiza (1). }\end{array}$ & 4 \\
\hline $\begin{array}{l}\text { Estudios cualitativos } \\
\text { exploratorios }\end{array}$ & $\begin{array}{l}\text { Australia (1), Inglaterra (1), multinacional } \\
\text { (1). }\end{array}$ & 3 \\
\hline
\end{tabular}


Estudios cuantitativos descriptivos

En la Tabla 3 se presentan los estudios de tipo cuantitativo-descriptivo, donde analizamos principalmente cómo se ha medido la resiliencia en población con diabetes tipo 2, y qué resultados se obtienen de la asociación con otras variables.

Aquí se muestra que el instrumento más utilizado es la Escala de Resiliencia de Connor-Davidson, CD-RISC, en sus dos versiones de 25 y 2 items ( $n=5$; 33.33\%), le sigue la Escala de Resiliencia de Wagnild-Young ( $n=2$; 13.33\%), aparecen otras dos escalas Annalakshmi Resilience ScaleForma A y State-Trait Resilience Inventory $(n=2$; $13.33 \%)$ con una mención cada una (Chakraborty y Chaudhury, 2015; Nawaz, Malik y Batool, 2014), y se observa que el resto de los estudios ( $n=6 ; 40 \%$ ) no mide la resiliencia directamente sino a través de otras variables (véase Tabla 3 ).

Por otro lado, la variable asociada más medida en estos estudios es la hemoglobina glucosilada $(n=4)$, encontrando que tiene una significativa correlación negativa con la resiliencia (Démden et al., 2017; DeNisco, 2011; Steinhardt et al., 2015a; 2015b; Yi, Vitaliano, Smith, Yi, y Weinger, 2008); le siguen variables como la depresión $(n=3)$, el estrés emocional $(n=3)$, el estado de salud general $(n=3)$, la calidad de vida $(n=2)$, el afrontamiento $(n=2)$, el optimismo $(n=2)$, el apoyo social $(n=2)$, y el autocuidado $(n=2)$, entre otras (que cuentan con una sola mención). También se puede ver en la Tabla 3 que en algunos estudios se mide una sola variable, así como en otros se miden varias a la vez. 
Tabla 3. Resultados de los estudios cuantitativos-descriptivos que miden la resiliencia y otras variables en la diabetes tipo 2 (parte 1 de 4)

\begin{tabular}{|c|c|c|c|c|}
\hline Autores & Población & $\begin{array}{l}\text { Medición de } \\
\text { resiliencia }\end{array}$ & $\begin{array}{l}\text { Variables } \\
\text { asociadas }\end{array}$ & Resultados principales \\
\hline $\begin{array}{l}\text { Bahremand } \\
\text { et al. (2014). }\end{array}$ & $\begin{array}{l}225 \\
\text { participantes } \\
\text { adultos. }\end{array}$ & $\begin{array}{l}\text { Escala de } \\
\text { Resiliencia de } \\
\text { Connor- } \\
\text { Davidson (CD- } \\
\text { RISC, de 25 } \\
\text { items). }\end{array}$ & $\begin{array}{l}\text { Estrés } \\
\text { emocional, } \\
\text { funcionamient } \\
\text { o familiar. }\end{array}$ & $\begin{array}{l}\text { Los altos niveles de resiliencia } \\
\text { resultan en un mejoramiento de la } \\
\text { salud mental y reducen el alto } \\
\text { resgo de trastomos psicológicos en } \\
\text { el paciente con diabetes } 2 \text {, } \\
\text { contrariamente, bajos niveles de } \\
\text { resiliencia están asociados con una } \\
\text { disminución en la salud mental de } \\
\text { estos pacientes. La resiliencia juega } \\
\text { un rol mediador entre el } \\
\text { funcionamiento familiar y la salud } \\
\text { mental. }\end{array}$ \\
\hline $\begin{array}{l}\text { Chakraborty } \\
\text { y } \\
\text { Chaudhury } \\
\text { (2015). }\end{array}$ & $\begin{array}{l}140 \text { hombres } \\
\text { de } 45-55 \\
\text { años. }\end{array}$ & $\begin{array}{l}\text { Annalakshmi } \\
\text { Resilience } \\
\text { Scale (Forma } \\
\text { A). }\end{array}$ & $\begin{array}{l}\text { Calidad de } \\
\text { vida, felicidad, } \\
\text { afrontamiento. }\end{array}$ & $\begin{array}{l}\text { La felicidad fue el factor que } \\
\text { contribuyó más comúnmente a la } \\
\text { calidad de vida (asociada a } \\
\text { resiliencia) tanto en grupos } \\
\text { enfermos como sin enfermedad. }\end{array}$ \\
\hline $\begin{array}{l}\text { Démden et } \\
\text { al. (2017). }\end{array}$ & $\begin{array}{l}151 \\
\text { participantes } \\
\text { de18-25 } \\
\text { años. }\end{array}$ & $\begin{array}{l}\text { CD-RISC (de } 2 \\
\text { items). }\end{array}$ & 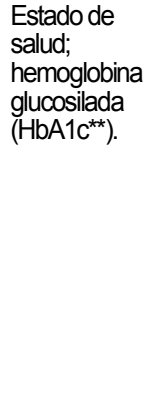 & $\begin{array}{l}\text { La forma agravada de la diabtes } \\
\text { está relacionada al éstres } \\
\text { emocional. Se reportan dificultades } \\
\text { en el manejo de los costos para los } \\
\text { cuidados. El reporte de trastomos } \\
\text { de salud mental es alto y bajo el } \\
\text { reporte de bienestar. Los trastomos } \\
\text { de la conducta alimentaria también } \\
\text { son significativos. La población } \\
\text { muestra baja resiliencia y bajo } \\
\text { control glicémico. Todos estos } \\
\text { factores se pueden evaluar con la } \\
\text { herramienta PAID (Áreas de } \\
\text { Problemas en la Diabetes). }\end{array}$ \\
\hline $\begin{array}{l}\text { DeNisco } \\
\text { (2011). }\end{array}$ & $\begin{array}{l}71 \text { mujeres } \\
\text { de 35-85 } \\
\text { años. }\end{array}$ & $\begin{array}{l}\text { Escala de } \\
\text { Resiliencia de } \\
\text { Wagnild- } \\
\text { Young. }\end{array}$ & $\mathrm{HbA1c}$. & $\begin{array}{l}\text { Los niveles de HbA1c y resiliencia } \\
\text { tuvieron una significativa correlación } \\
\text { negativa, con puntuación alta en } \\
\text { resiliencia mostraban niveles más } \\
\text { bajos de HbA1c. }\end{array}$ \\
\hline $\begin{array}{l}\text { Lynch, } \\
\text { Waite y } \\
\text { Davey } \\
\text { (2013). }\end{array}$ & $\begin{array}{l}801 \\
\text { participantes } \\
\text { adultos. }\end{array}$ & $\begin{array}{l}\text { No se mide la } \\
\text { resiliencia } \\
\text { directamente. }\end{array}$ & $\begin{array}{l}\text { Experiencias } \\
\text { de trauma en } \\
\text { la niñez } \\
\text { (reportadas } \\
\text { por los } \\
\text { pacientes). }\end{array}$ & $\begin{array}{l}\text { El } 50 \% \text { de pacientes estudiados } \\
\text { reportaron } 4 \text { o más experiencias } \\
\text { traumáticas en la niñez confirmando } \\
\text { que el trauma es central en el } \\
\text { desarrollo de diabetes tipo } 2 \text {. Los } \\
\text { resultados tienen implicación para } \\
\text { comenzar a trabajar con el enfoque } \\
\text { de la resiliencia en edades } \\
\text { tempranas. }\end{array}$ \\
\hline
\end{tabular}


Tabla 3. Resultados de los estudios cuantitativos-descriptivos que miden la resiliencia y otras variables en la diabetes tipo 2 (parte 2 de 4)

\begin{tabular}{|c|c|c|c|c|}
\hline Autores & Población & $\begin{array}{l}\text { Medición de } \\
\text { resiliencia }\end{array}$ & $\begin{array}{l}\text { Variables } \\
\text { asociadas }\end{array}$ & Resultados principales \\
\hline $\begin{array}{l}\text { Martín, } \\
\text { González, } \\
\text { Papín y } \\
\text { Suárez } \\
\text { (2018). }\end{array}$ & $\begin{array}{l}206 \\
\text { participantes } \\
\text { adultos. }\end{array}$ & $\begin{array}{l}\text { No se mide la } \\
\text { resiliencia } \\
\text { directamente. }\end{array}$ & $\begin{array}{l}\text { Adherencia } \\
\text { terapéutica (a } \\
\text { través del test } \\
\text { Motiv.Diaf- } \\
\text { DM2* que } \\
\text { mide } \\
\text { adherencia a } \\
\text { la dieta y } \\
\text { actividad física } \\
\text { en DM2*). }\end{array}$ & $\begin{array}{l}\text { El uso de este test bidimensional } \\
\text { mostró una buena confiabilidad y } \\
\text { validez. Las puntuaciones de la } \\
\text { segunda dimensión (actividad } \\
\text { física) convergen con las } \\
\text { puntuaciones de las necesidades } \\
\text { psicológicas básicas, resiliencia y } \\
\text { hemoglobina glucosilada. }\end{array}$ \\
\hline $\begin{array}{l}\text { Mertens, } \\
\text { Bosma, } \\
\text { Groffeny } \\
\text { van Eijk } \\
(2011) .\end{array}$ & $\begin{array}{l}361 \\
\text { participantes } \\
\text { de } 60 \text { y más } \\
\text { años. }\end{array}$ & $\begin{array}{l}\text { No se mide la } \\
\text { resiliencia } \\
\text { directamente. }\end{array}$ & $\begin{array}{l}\text { Depresión, } \\
\text { funcionamient } \\
\text { o social. }\end{array}$ & $\begin{array}{l}\text { Un alto nivel de destrezas está } \\
\text { significativamente asociado con el } \\
\text { funcionamiento físico, mental y } \\
\text { social (se describe como } \\
\text { resiliencia). Los altos niveles de } \\
\text { apoyo social e ingresos también } \\
\text { contribuyen significativamente para } \\
\text { tener éxito en el funcionamiento } \\
\text { social en esta población. }\end{array}$ \\
\hline $\begin{array}{l}\text { Nawaz, } \\
\text { Malik y } \\
\text { Batool } \\
\text { (2014). }\end{array}$ & $\begin{array}{l}242 \\
\text { participantes } \\
\text { adultos, } 108 \\
\text { mujeres y } \\
134 \\
\text { hombres. }\end{array}$ & $\begin{array}{l}\text { State-Trait } \\
\text { Resilience } \\
\text { Inventory. }\end{array}$ & $\begin{array}{l}\text { Calidad de } \\
\text { vida. }\end{array}$ & $\begin{array}{l}\text { La característica de la resiliencia } \\
\text { predice todos los aspectos de la } \\
\text { calidad de vida de los pacientes con } \\
\text { diabetes y explica } 17 \% \text { de varianza } \\
\text { en el funcionamiento físico, } 29 \% \text { en } \\
\text { funcionamiento psicológico, } 17 \% \\
\text { en funcionamiento ambiental y } 30 \\
\% \text { en la dimensión social. El largo } \\
\text { estadío de vivir con diabetes puede } \\
\text { beneficiarse a través de una } \\
\text { intervención direccionada a trabajar } \\
\text { la resiliencia. }\end{array}$ \\
\hline $\begin{array}{l}\text { Perna et al. } \\
\text { (2013). }\end{array}$ & $\begin{array}{l}3942 \\
\text { participantes } \\
\text { de } 64 \text { y más } \\
\text { años. }\end{array}$ & $\begin{array}{l}\text { Escala de } \\
\text { Resiliencia de } \\
\text { Wagnild-Young } \\
\text { (versión corta- } \\
\text { RS11). }\end{array}$ & $\begin{array}{l}\text { Neuropatía } \\
\text { diabética, } \\
\text { nivel } \\
\text { educativo. }\end{array}$ & $\begin{array}{l}\text { Se encontró que entre los } \\
\text { participantes con bajo nivel } \\
\text { educativo y altos niveles de } \\
\text { resiliencia se tuvo baja probabilidad } \\
\text { de suffrir neuropatía, en } \\
\text { comparación con los que tuvieron } \\
\text { baja resiliencia. Los pacientes con } \\
\text { neuropatía diabética muestran baja } \\
\text { resiliencia e incrementa el riesgo } \\
\text { entre los individuos adultos } \\
\text { mayores con bajo nivel educativo. }\end{array}$ \\
\hline
\end{tabular}


Tabla 3. Resultados de los estudios cuantitativos-descriptivos que miden la resiliencia y otras variables en la diabetes tipo 2 (parte 3 de 4)

\begin{tabular}{|c|c|c|c|}
\hline Autores & Población & $\begin{array}{c}\text { Medición de } \\
\text { resiliencia }\end{array}$ & $\begin{array}{c}\text { Variables } \\
\text { asociadas }\end{array}$ \\
\hline $\begin{array}{l}\text { Rendón y } \\
\text { Lugli (2013). }\end{array}$ & $\begin{array}{l}300 \\
\text { participantes } \\
\text { adultos, } 103 \\
\text { hombres y } \\
197 \text { mujeres. }\end{array}$ & $\begin{array}{l}\text { No se mide la } \\
\text { resiliencia } \\
\text { directamente. }\end{array}$ & $\begin{array}{l}\text { Afrontamiento } \\
\text {, optimismo, } \\
\text { apoyo social, } \\
\text { redes de } \\
\text { apoyo. }\end{array}$ \\
\hline $\begin{array}{l}\text { Speight, } \\
\text { Browne, } \\
\text { Holmes- } \\
\text { Truscott, } \\
\text { Hendrieckx } \\
\text { y Pouwer } \\
\text { (2012). }\end{array}$ & $\begin{array}{l}3,338 \\
\text { participantes } \\
\text { adultos. }\end{array}$ & $\begin{array}{l}\text { No se mide la } \\
\text { resiliencia } \\
\text { directamente. }\end{array}$ & $\begin{array}{l}\text { Depresión, } \\
\text { creencias en } \\
\text { salud, apoyo } \\
\text { social, } \\
\text { autocuidado, } \\
\text { empoderamie } \\
\text { nto, otros. }\end{array}$ \\
\hline
\end{tabular}

\section{Resultados principales}

El tipo de diabetes no afecta el apoyo social percibido, pero sí a través de relaciones mediadas por el optimismo y las estrategias de afrontamiento; destacando que son éstas últimas las que determinan el apoyo social de los sujetos. El optimismo está asociado a la variable resiliencia, los sujetos optimistas emplearon mayor estrategia de aproximación al problema y esto conllevó a tener mayores redes sociales y apoyo social percibido (más resiliencia).

Diabetes y depresión se relacionan como los mejores amigos. Se perciben deficiencias en el sistema de salud actual. Existe asociación entre el estatus en salud y las creencias en salud, apoyo social, autocuidado y empoderamiento. Aspectos positivos de la salud mental como la resilienicia, la autoestima y el optimismo, son importantes mediadores de los resultados en diabetes.

\begin{tabular}{|c|c|c|c|}
\hline $\begin{array}{l}\text { Steinhardt } \\
\text { et al. } \\
\text { (2015b). }\end{array}$ & $\begin{array}{l}93 \\
\text { participantes, } \\
62 \text { mujeres y } \\
31 \text { hombres } \\
\text { de 31-85 } \\
\text { años. }\end{array}$ & $\begin{array}{l}\text { CD-RISC (de } \\
25 \text { ítems). }\end{array}$ & $\begin{array}{l}\text { Crecimiento } \\
\text { personal, } \\
\text { depresión, } \\
\text { HbA1c. }\end{array}$ \\
\hline
\end{tabular}

Velthorst, 811 Reichenber 811 g, de 34-44 Rabinowitz años. y Levine (2015).
Quienes tienen más crecimiento personal tienen más altos niveles de resiliencia y pocos síntomas de depresión, así como baja HbA1c, mientras que los individuos deprimidos registran una baja resiliencia y altos niveles de síntomas depresivos, así como alta $\mathrm{HbA1c}$.

Se reportan problemas de salud comparables a los que reporta la ( salud, sin embargo, también reportan ansiedad y estrés. Aún no se valoran todas las vaniables pues para observar su comportamiento

\begin{tabular}{|c|c|c|}
\hline $\begin{array}{l}\text { CD-RISC (de } 2 \\
\text { items). }\end{array}$ & $\begin{array}{l}\text { Estado de } \\
\text { salud, } \\
\text { autopercepció } \\
\text { n en salud, } \\
\text { ansiedad, } \\
\text { estrés } \\
\text { emocional. }\end{array}$ & $\begin{array}{l}\text { Se reportan problemas de salud } \\
\text { comparables a los que reporta la } \\
\text { OMS a nivel mundial. La mayoría } \\
\text { tiene una autopercepción de buena } \\
\text { salud, sin embargo, también } \\
\text { reportan ansiedad y estrés. Aún no } \\
\text { se valoran todas las variables pues } \\
\text { es un estudio longitudinal a } 10 \text { años } \\
\text { para observar su comportamiento } \\
\text { resiliente. }\end{array}$ \\
\hline
\end{tabular}


Tabla 3. Resultados de los estudios cuantitativos-descriptivos que miden la resiliencia y otras variables en la diabetes tipo 2 (parte 4 de 4)

\begin{tabular}{|c|c|c|c|c|}
\hline Autores & Población & $\begin{array}{l}\text { Medición de } \\
\text { resiliencia }\end{array}$ & $\begin{array}{c}\text { Variables } \\
\text { asociadas }\end{array}$ & Resultados principales \\
\hline $\begin{array}{l}\text { Willich, } \\
\text { Guerreiro y } \\
\text { Hegadoren } \\
\text { (2016). }\end{array}$ & $\begin{array}{l}\text { BL603 } \\
\text { participantes } \\
\text { adultos. }\end{array}$ & $\begin{array}{l}\text { CD-RISC (de } \\
25 \text { ítems). }\end{array}$ & $\begin{array}{l}\text { Estado de } \\
\text { salud. }\end{array}$ & $\begin{array}{l}\text { Los participantes con enfermedad } \\
\text { renal mostraron menor resiliencia } \\
\text { que las personas con diabetes. El } \\
\text { tipo de enfermedad crónica, la } \\
\text { duración de la enfermedad, el IMC } \\
\text { (índice de masa corporal) y las } \\
\text { creencias religiosas influyeron en la } \\
\text { resiliencia mostrada. }\end{array}$ \\
\hline $\begin{array}{l}\text { Yi, Vitaliano, } \\
\text { Smith, Yi, y } \\
\text { Weinger } \\
\text { (2008). }\end{array}$ & $\begin{array}{l}111 \\
\text { participantes } \\
\text { de 18 a } 75 \\
\text { años. }\end{array}$ & $\begin{array}{l}\text { No se mide la } \\
\text { resiliencia } \\
\text { directamente. }\end{array}$ & $\begin{array}{l}\text { Optimismo, } \\
\text { autoestima, } \\
\text { autoeficacia, } \\
\text { autodestreza, } \\
\text { autocuidado, } \\
\text { estrés } \\
\text { emocional, } \\
\text { HbA1c. }\end{array}$ & $\begin{array}{l}\text { La resiliencia y su interacción con el } \\
\text { estrés emocional relacionado a la } \\
\text { diabetes predicen la salud física } \\
\text { (HbA1c) al cabo de un año. Los } \\
\text { pacientes con moderada o baja } \\
\text { resiliencia mostraron una fuerte } \\
\text { asociación entre el estrés } \\
\text { alcanzado y el empeoramiento de } \\
\text { la HbA1c a través del tiempo. La } \\
\text { baja resiliencia también estuvo } \\
\text { asociada con pocas conductas de } \\
\text { autocuidado y un incremento del } \\
\text { estrés emocional. }\end{array}$ \\
\hline
\end{tabular}

${ }^{*}$ DM2 $=$ Diabetes Mellitus tipo 2.

**HbAlc = Hemoglobina glucosilada

Los resultados principales de estos estudios abonan a la efectividad de contar con altos niveles de resiliencia para lograr mayor control del padecimiento y mejorar la calidad de vida de la población adulta con diabetes tipo 2.

Estudios sobre intervenciones clinicas

Los estudios que desarrollan intervenciones para potenciar la resiliencia en adultos con diabetes, se incluyen en la Tabla 4. El diseño de las intervenciones es variado, pero en promedio tuvieron una duración de entre tres y seis meses. Seis estudios $(75 \%)$ contaron con un diseño experimental o cuasiexperimental con grupo control pretest-postest (Bradshaw et al., 2007; 2008; Hakimian, 
Shahreza y Hazar, 2016; Pyatak et al., 2016; 2018; Steinhardt et al., 2015a; 2015b;), una de las intervenciones (12.5\%) fue un estudio piloto (Steindhardt, Mamerow, Brown y Jolly, 2009) y otra más (12.5\%) sólo fue de promoción de la resiliencia (Jeffries-Stokes, Stokes y McDonald, 2015).

En cuanto a la medición de la variable diabetes tipo 2, cinco estudios reportan la toma de la hemoglobina glucosilada (Bradshaw et al., 2007; 2008; Pyatak et al., 2016; 2018; Steinhardt, Brown, Dubois, Harrison, Lehrer y Jaggars, 2015; otros tres aplicaron cuestionarios de conocimiento y/ o empoderamiento en diabetes (Jeffries-Stokes et al., 2015; Steindhardt et al., 2009; 2015), y dos recurrieron a la medición de glucosa en ayunas (Hakimian et al., 2016; Steinhardt et al., 2015), nótese que un estudio incluyó las tres mediciones señaladas (Steinhardt et al., 2015).

Los resultados de las intervenciones muestran que en todas las que se midió el conocimiento y/o empoderamiento de la diabetes, éste aumentó (aunque no alcanzó significancia estadística), y en las que se midió el azúcar en ayunas y la hemoglobina glucosilada disminuyeron los parámentros, pero sólo dos alcanzaron una significancia de $p<.01$ (Hakimian et al., 2016; Pyatak et al., 2018) con respecto al grupo control.

En cuestión de la medición de la resiliencia, cuatro estudios (50\%) la midieron a través de test de elaboración propia (Bradshaw et al., 2007; 2008; Pyatak et al., 2016; 2018), tres más (37.5\%) utilizaron el instrumento CD-RISC de Connor-Davidson (Hakimian et al., 2016; Steindhardt et al,, 2009; 2015, y uno último (12.5\%) sólo atendió a la resiliencia como factor de prevención sin medirla (Jeffries-Stokes et al., 2015). 
Tabla 4. Resultados de los estudios sobre intervenciones para potenciar la resiliencia en población con diabetes (parte 1 de 2).

\begin{tabular}{|c|c|c|c|}
\hline Autores & Población & $\begin{array}{c}\text { Instrumentos de } \\
\text { medición }\end{array}$ & Intervención \\
\hline $\begin{array}{l}\text { Bradshaw et } \\
\text { al. (2007). }\end{array}$ & $\begin{array}{l}67 \text { participantes, } 37 \\
\text { con tratamiento usual y } \\
30 \text { con clases de } \\
\text { resiliencia. }\end{array}$ & $\begin{array}{l}\text { Se realizaron medidas } \\
\text { fisiológicas (toma de } \\
\text { HbA1c') y psicológicas } \\
\text { a través de varios } \\
\text { cuestionarios del } \\
\text { Michigan Diabetes } \\
\text { Research and Training } \\
\text { Center, los cuales } \\
\text { habían sido validados y } \\
\text { poseían una } \\
\text { confiabilidad aceptable. }\end{array}$ & $\begin{array}{l}\text { La intervención se llevó a cabo } \\
\text { en } 10 \text { módulos con un total de } \\
15 \text { horas. Cada módulo trató } \\
\text { sobre dimensiones de la } \\
\text { resiliencia y se trabajaron } \\
\text { herramientas para fortalecer la } \\
\text { mente, el cuerpo y el espírtu. }\end{array}$ \\
\hline $\begin{array}{l}\text { Bradshaw et } \\
\text { al. (2008). }\end{array}$ & $\begin{array}{l}67 \text { personas que } \\
\text { previamente habian } \\
\text { recibido una } \\
\text { autoeducación en } \\
\text { diabetes de tipo } \\
\text { estándar. }\end{array}$ & $\begin{array}{l}\text { Este estudio es la } \\
\text { continuación a seis } \\
\text { meses del de } \\
\text { Bradshaw et al. (2007), } \\
\text { por lo que se midieron } \\
\text { las mismas variables. }\end{array}$ & $\begin{array}{l}\text { Aplicar un enfoque en } \\
\text { resiliencia en conjunto con un } \\
\text { programa de educación en } \\
\text { diabetes de tipo estándar, para } \\
\text { apoyar a los pacientes a llegar a } \\
\text { ser más autodirectivos en los } \\
\text { cuidados de su diabetes. }\end{array}$ \\
\hline $\begin{array}{l}\text { Hakimian, } \\
\text { Shahreza y } \\
\text { Hazar (2016). }\end{array}$ & $\begin{array}{l}62 \text { adultos divididos } \\
\text { aleatoriamente en } \\
\text { grupo control y } \\
\text { experimental. }\end{array}$ & $\begin{array}{l}\text { Se midió el azúcar en } \\
\text { ayunas, y postpandrial; } \\
\text { se aplicó la CD-RISC } \\
\text { adaptada a población } \\
\text { de lrán. }\end{array}$ & $\begin{array}{l}\text { Programa de } 12 \text { sesiones } \\
\text { semanales de educación en el } \\
\text { autocontrol de la diabetes. } \\
\text { Donde, además de tratar la } \\
\text { parte física de la enfermedad, } \\
\text { se enseñaron diversas } \\
\text { herramientas psicológicas. }\end{array}$ \\
\hline $\begin{array}{l}\text { Jeffries- } \\
\text { Stokes, } \\
\text { Stokes y } \\
\text { McDonald } \\
\text { (2015). }\end{array}$ & $\begin{array}{l}\text { Comunidades } \\
\text { aborígenes de } \\
\text { Australia. Participaron } \\
\text { más de } 2,500 \\
\text { personas ( } 58 \% \text { de la } \\
\text { población). }\end{array}$ & $\begin{array}{l}\text { Se realizaron } \\
\text { valoraciones clínicas } \\
\text { para detección de } \\
\text { diabetes o enfermedad } \\
\text { renal. }\end{array}$ & $\begin{array}{l}\text { La intervención consistió en } \\
\text { enseñar a los participantes a } \\
\text { través de "Comunidades de } \\
\text { Arte", los factores de riesgo para } \\
\text { enfermedad renal y diabetes, } \\
\text { herramientas para la resolución } \\
\text { de problemas y formas de } \\
\text { expresarse y defenderse dentro } \\
\text { de su comunidad. }\end{array}$ \\
\hline $\begin{array}{l}\text { Pyatak et al. } \\
\text { (2016). }\end{array}$ & $\begin{array}{l}81 \text { adultos de entre } 18- \\
30 \text { años, con diabetes } \\
1 \text { ó } 2 \text { y una HbA1c }> \\
8 \% \text { al inicio del estudio. } \\
\text { Todos los participantes } \\
\text { fueron de diversas } \\
\text { etnias y con un bajo } \\
\text { estatus } \\
\text { socioeconómico. }\end{array}$ & $\begin{array}{l}\text { Se midió la HbA1c, y se } \\
\text { utilizó el Dependent } \\
\text { Quality of Life (ADD- } \\
\text { QoL, de } 36 \text { reactivos) } \\
\text { para asociar la } \\
\text { resiliencia, se hicieron } \\
\text { varias mediciones más } \\
\text { al inicio y a los } 6 \text { meses }\end{array}$ & $\begin{array}{l}\text { Los participantes del grupo } \\
\text { control recibieron un manual } \\
\text { que constaba de } 7 \text { módulos, y } \\
\text { se les llamaba cada quincena } \\
\text { para resolver dudas. El grupo } \\
\text { intervenido recibió } 12 \text { sesiones } \\
\text { quincenales individuales } \\
\text { durante } 6 \text { meses, en cada } \\
\text { módulo se incluian metas } \\
\text { personales. }\end{array}$ \\
\hline
\end{tabular}


Tabla 4. Resultados de los estudios sobre intervenciones para potenciar la resiliencia en población con diabetes (parte 2 de 2).

\begin{tabular}{|c|c|c|c|}
\hline Autores & Población & $\begin{array}{l}\text { Instrumentos de } \\
\text { medición }\end{array}$ & Intervención \\
\hline $\begin{array}{l}\text { Pyatak et al. } \\
\text { (2018). }\end{array}$ & $\begin{array}{l}81 \text { adultos jóvenes con } \\
\text { diversidad étnica } \\
\text { fueron aleatorizados } \\
\text { para grupo control o } \\
\text { experimental. }\end{array}$ & $\begin{array}{l}\text { Se midió la HbA1c, y se } \\
\text { aplicó una encuesta } \\
\text { elaborada que medía } \\
\text { datos demográficos, } \\
\text { clíicos y características } \\
\text { psicológicas. }\end{array}$ & $\begin{array}{l}\text { Intervención manualizada } \\
\text { hecha a la talla de cada } \\
\text { persona en el transcurso de } 6 \\
\text { meses, con promedio de } 10-16 \\
\text { sesiones llevadas a cabo en el } \\
\text { hogar o comunidad de origen. }\end{array}$ \\
\hline $\begin{array}{l}\text { Steindhardt, } \\
\text { Mamerow, } \\
\text { Brown y Jolly } \\
\text { (2009). }\end{array}$ & $\begin{array}{l}\text { Iniciaron } 8 \text { hombres y } 8 \\
\text { mujeres y teminaron } \\
\text { el programa sólo } 6 \\
\text { hombres y } 6 \text { mujeres. }\end{array}$ & $\begin{array}{l}\text { La resiliencia se midió } \\
\text { con la CD-RISC de } 25 \\
\text { ítems, y el } \\
\text { empoderamiento en } \\
\text { diabetes se midió con el } \\
\text { Diabetes } \\
\text { Empowement Scale } \\
\text { (DES-28 ítems). }\end{array}$ & $\begin{array}{l}\text { El programa se llevó a cabo en } \\
4 \text { semanas, dando la } \\
\text { intervención en resiliencia en la } \\
\text { primera hora y en la segunda, } \\
\text { se trataron los aspectos de } \\
\text { autocontrol en diabetes. } \\
\text { Además de } 8 \text { reuniones } \\
\text { quincenales (de 1:30 horas) } \\
\text { para retroalimentación. }\end{array}$ \\
\hline $\begin{array}{l}\text { Steinhardt, } \\
\text { Brown, } \\
\text { Dubois, } \\
\text { Harrison, } \\
\text { Lehrer y } \\
\text { Jaggars } \\
\text { (2015). }\end{array}$ & $\begin{array}{l}65 \text { adultos, un grupo } \\
\text { experimental de } 32 \text { y } \\
\text { uno control de } 33 \text {. }\end{array}$ & $\begin{array}{l}\text { Se midió el } \\
\text { conocimiento en } \\
\text { diabetes, bienestar } \\
\text { psicológico (incluyó la } \\
\text { resiliencia), autocontrol } \\
\text { en diabetes, } \\
\text { marcadores fisiológicos, } \\
\text { además de la glucosa } \\
\text { en ayunas y la HbA1c. } \\
\text { La resiliencia se midió } \\
\text { con la CD-RISC. }\end{array}$ & $\begin{array}{l}\text { Los grupos control recibieron } \\
\text { una educación estandarizada } \\
\text { en diabetes y los grupos } \\
\text { experimentales, además de } \\
\text { ésta, recibieron la enseñanza } \\
\text { sobre resiliencia. }\end{array}$ \\
\hline
\end{tabular}

${ }^{*} \mathrm{HbAlc}=$ Hemoglobina glucosilada.

Los resultados sobre la medición de la resiliencia arrojan que tres estudios (Bradshaw et al., 2008; Hakimian et al., 2016; Pyatak et al., 2018) alcanzaron una significancia de $p$ $<$.05. También se midió la calidad de vida (bienestar psicológico), asociándola con la resiliencia en un estudio (Pyatak et al., 2018) que reportó un aumento significativo con respecto al grupo control $(p<.04)$. Los demás estudios (50\%) no reportaron significancia estadística. 
Estudios sobre modelos de resiliencia y revisiones teóricas

Encontramos dos estudios que trabajan sobre modelos para generar mayor resiliencia en las personas con diabetes. Klein y Lippa (2012) asumen en su estudio que la diabetes es un padecimiento de cambios dinámicos y que requiere un Análisis de la Tarea Cognitiva (CTA, por sus siglas en inglés) donde se pueda saber cómo entiende el paciente su enfermedad y cómo enfrenta las demandas para su manejo, por lo tanto, se establece que al paciente le funcionará seguir un modelo de control a través del cual se estructuren sus actividades y decisiones para el manejo de su glucosa, y esto lo llevará a adquirir mayor resiliencia, pudiendo detectar problemas, identificar recursos, monitorearse e interpretar sus resultados.

El otro modelo es de tipo ecológico (Aldwin y Igarashi, 2012) y concibe a la resiliencia como un fenómeno que se da a nivel individual, contextual y sociocultural. Este modelo ecológico transaccional está basado en el estrés individual y los procesos de afrontamiento, los cuales son informados como recursos y barreras en los niveles contextual y sociocultural. La aplicación del modelo en problemas crónicos de salud como la diabetes, ha demostrado que las intervenciones requieren del trabajo en los tres niveles.

Por otro lado, encontramos dos estudios que son revisiones teóricas breves enfocadas a analizar cómo influyen las respuestas psicobiológicas en el desarrollo de la diabetes de tipo 2 (Ehlert, 2013; Pervanidou, Agorastos, Kolaitis y Chrousos, 2017).

Ambas revisiones teóricas investigan sobre los estresores tempranos de la vida (experiencias traumáticas de la niñez), observan que el estrés se asocia con la activación del eje hipotalámico-adrenal-pituitario y la 
exitación del sistema nervioso simpático, y llevan al individuo a una elevación de cortisol y catecolaminas, por lo que las experiencias de estrés crónico y/o intenso representan dishomeostasis (también llamada alostasis), la cual se relaciona con mayor morbilidad, pudiendo desarrollar obesidad, síndrome metabólico, diabetes tipo 2 , arteriosclerosis, osteoporosis y disfunción inmune (Pervanidou y Chrouss, 2012); y entre los desórdenes psiquiátricos el más común es el síndrome de estrés postraumático (Ehlert, 2013). Los periodos críticos del desarrollo cerebral representan ventanas de tiempo para elevar la plasticidad sináptica, tener mediadores de la vulnerabilidad o aumentar la resiliencia (Pervanidou et al., 2017).

\section{Estudios cualitativos exploratorios}

Se encontraron tres estudios. En un uno, se valoran las experiencias psicosociales de la diabetes, incluyendo aspectos negativos y las maneras de afrontamiento (Stuckey et al., 2014), en este estudio, cerca de 50\% de la población estudiada reporta un bienestar psicosocial negativo en comparación con cerca de 30\% que lo reportan positivo; entre los aspectos negativos destacan la ansiedad, el miedo, la depresión y el humor negativo o desesperanza; entre los aspectos positivos están el tener una perspectiva positiva, tener resiliencia, y recibir apoyo psicosocial a través de familiares, amigos, personal de salud y otras personas con diabetes.

Otro estudio explora la incorporación de la espiritualidad en las personas con diabetes (Unantenne, Warren, Canaway y Manderson, 2011), y ubica cuatro dimensiones de espiritualidad y fe: 1) afrontamiento y apoyo; 2) aceptación; 3) sanación; y 4) salud-bienestar 
subjetivo; a través de las cuales el individuo se va adaptando a su enfermedad y genera mayor resiliencia.

En un estudio más, se explora el autocontrol de la diabetes tipo 2 que tienen las mujeres de diversos gupos étnicos (Majeed-Ariss, Jackson, Knapp y Cheater, 2015). Se asume que las prácticas culturales influyen de generación en generación para el autocontrol de la diabetes y la resiliencia en esta población; el estudio sugiere que los profesionales de la salud comprendan las influencias culturales para generar mayor autocontrol y resiliencia.

\section{CONCLUSIONES}

Resalta la productividad de estudios publicados en idioma inglés, así como de origen estadounidense, de acuerdo con Fernandes de Araújo y Bermúdez (2015) este hecho se relaciona con factores como la universalización del idioma, el mayor impacto de los artículos publicados en inglés, así como con mayores inversiones que hace el país destinadas a la investigación. En contraste, en la región de América Latina es escasa la producción de estudios de este tipo (sólo 6.66\%).

De acuerdo con los estudios analizados, se aduce que la resiliencia influye positivamente en el control glicémico de la población con diabetes tipo 2. Como lo señala la OMS (2016), la mayoría de los estudios midieron la hemoglobina glucosilada ya que es el método preferido para monitorear el control de la glucemia en la diabetes. La resiliencia en los adultos con diabetes tipo 2 se registra en menor medida cuando existe depresión, estrés emocional, baja percepción de calidad de vida y comorbilidad, principalmente; y se observa un mayor nivel de resiliencia y control glicémico cuando hay mayores puntuaciones de 
optimismo, afrontamiento directo, apoyo social y autocuidado, entre otras.

Los estudios sobre intervenciones parecen aún escasos a pesar de que se ha probado la efectividad de trabajar la resiliencia para la mejora del padecimiento (Hakimian et al., 2016; Pyatak et al., 2018) y el aumento de la variable como tal (Bradshaw et al., 2008; Hakimian et al., 2016; Pyatak et al., 2018), aunque en otras investigaciones no se reporta un aumento inmediato de la resiliencia sino hasta los seis meses (Bradshaw et al., 2007; 2008).

El instrumento más reportado para medir la resiliencia es la Escala de Resiliencia de Connor-Davidson (Bahremand et al., 2014; Démden et al., 2017; Hakimian et al., 2016; Steindhardt et al., 2009; 2015a; 2015b; Velthorst, Reichenberg, Rabinowitz y Levine, 2015; Willrich Guerreiro y Hegadoren, 2016). Otros estudios reportan la medición de resiliencia a través de instrumentos de elaboración propia y otros más no miden la variable directamente sino a través de otras variables (véase Tabla 3), y esto pareciera apuntar a que aún se sigue con el conflicto de no reconocer exactamente qué es la resiliencia o qué tanto puede abarcar.

Los modelos de resiliencia que se retoman en esta revisión dan cuenta de que se busca ampliar las intervenciones a aspectos tanto de tipo cognitivo como de tipo ecológico.

Se afirma también que los aspectos psicobiológicos que aparecen desde etapas muy tempranas de la vida al someter al individuo a eventos altamente estresantes, lo llevan a desarrollar trastornos psicológicos (tipo estrés postraumático), además de que influyen en el desarrollo de ciertas morbilidades como la diabetes tipo 2 (Ehlert, 2013; Lynch et al., 2013; Pervanidou et al., 2017), por lo que es importante trabajar con estas personas lo más 
inmediatamente posible en su resiliencia, evitando así en gran medida la aparición de enfermedades a largo plazo.

Los estudios de tipo cualitativo demuestran que la percepción de las personas derivada de factores religiosos y culturales influye de manera importante para el desarrollo de resiliencia y autocontrol de su diabetes, destacando la importancia del apoyo de sus grupos sociales de referencia.

Por último, esta revisión teórica tiene como limitación que sólo se recurrió a fuentes bibliográficas gratuitas y no se accedió a aquellas publicaciones que tenían algún costo, por lo que para futuras investigaciones de este tipo se sugiere considerar tener acceso a todas las publicaciones que hablen sobre el tema, ya sean libres o con costo, para tener un panorama mucho más completo.

\section{REFERENCIAS}

Aldwin, C., y Igarashi, H. (2012). An ecological model of resilience in late life. Annual Review of Gerontology and Geriatrics, 32(1), 115-130. doi:10.1891/0198-8794.32.115

Bahremand, M., Rai, A., Alikhani, M., Mohammadi, S.,

Shahebrahimi, K. y Janjani, P. (2014). Relationship between family functioning and mental health considering the mediating role of resiliency in type 2 diabetes mellitus patients. Global Journal of Health Science, 7(3), 254-259. doi:10.5539/gihs.v7n3p254

Bradshaw, B. G. et al. (2007). Determining the efficacy of a resiliency training approach in adults with type 2 diabetes. The Diabetes Educator, 33(4), 650-659. doi:10.1177/0145721707303809

Bradshaw, B. G., Richardson, G. E., Kumpfer, K., Carlson, J., Kulkarni, K. y Brooks, A. (2008). The role of the registered 
dietitian: Enhancing positive outcomes using a resiliency training approach in people with type 2 diabetes. Journal of the American Dietetic Association, 108(9), A51.

doi:10.1016/j.jada.2008.06.097

Brix, C., Schleußner, C., Füller, J., Röhrig, B., Wendt, T. y Strauß, B. (2008). The need for psychosocial support and its determinants in a sample of patients undergoing radiooncological treatment of cancer. Journal of Psychosomatic Research, 65(6), 541-548. doi:10.1016/j.jpsychores.2008.05.010

Chakraborty, D. y Chaudhury, A. (2015). A study on quality of life of people with chronic illness. Indian Journal of Health and Wellbeing, 6(12), 1165-1168. Recuperado de http://www.aahrw.com/index.php/home/journal detail/19\# $\underline{\text { list }}$

Démden, H. et al. (2017). Psychosocial screening and management of young people aged 18-25 years with diabetes. Internal Medicine Journal, 47(4), 415-423. doi:10.1111/ imj.13375

DeNisco, S. (2011). Exploring the relationship between resilience and diabetes outcomes in African Americans. Journal of the American Academy of Nurse Practioners, 23(11), 602-610. doi:10.1111/j.1745-7599.2011.00648.x

Ehlert, U. (2013). Enduring psychobiological effects of childhood adversity. Psychoneuroendocrinology, 38, 1850-1857. doi:10.1016/j.psyneuen.2013.06.007

Fernandes de Araújo, L. y Bermúdez, M. de la P. (2015). Resiliencia en adultos: una revisión teórica. Terapia psicológica, 33(3), 257-276. doi:10.4067/S071848082015000300009

García-Vesga, M. y Domínguez-de la Ossa, E. (2013). Desarrollo teórico de la resiliencia y su aplicación en situaciones adversas: Una revisión analítica. Revista Latinoamericana de 
Ciencias Sociales, Niñez y Juventud, 11(1), 63-77.

doi:10.11600/1692715x.1113300812

Hakimian,M., Shahreza, F. y Hazar, N. (2016). Self-management education and its association with resilience in diabetic patients. Iranian Journal of Diabetes and Obesity, 7(4), 141-145. Recuperado de http://ijdo.ssu.ac.ir/article-1-263-en.pdf Jamison, M., Weidner, A., Romero, A. y Amundsen, C. (2007).

Lack of psychological resilience: an important correlate 5 for urinary incontinence. International Urogynecology Journal, 18(10), 1127-1132. doi:10.1007/s00192-007-0315-y

Jeffries-Stokes C., Stokes A. y McDonald, L. (2015). Pulkurlkpathe western desert kidney health project strategies to build resilience and combat kidney disease and type 2 diabetes (wdkhp). Internal Medicine Journal, (Abstracts), 45, 12. doi:10.1111/imj.12782 44

Klein, H.A. y Lippa, K. D. (2012). Assuming control after system failure: type II diabetes self-management. Cognition, Technology \& Work, 14(3), 243-251. doi:10.1007/s10111-0110206-3

Kralik, D., Van Loon, A. y Visentin, K. (2006). Resilience in the chronic illness experience. Educational Action Research, 14(2), 187-201. doi:10.1080/09650790600718035

Lynch, L., Waite, R. y Dave, M. P. (2013). Adverse childhood experiences and diabetes in adulthood. Support for a Collaborative Approach to Primary Care, doi:10.1007/s10591013-9262-6

Majeed-Ariss, R., Jackson, C., Knapp, P. y Cheater, F. M. (2015). British-Pakistani women's perspectives of diabetes selfmanagement: the role of identity. Journal of Clinical nursing, 24(17-18), 2571-2580. doi:10.1111/jocn.12865

Martín, R., González, X., Papín, C. y Suárez, J. (2018).

Development and validation of a questionnaire for assessing the characteristics of diet and physical activity 
in patients with type 2 diabetes. Psicothema.

doi:10.7334/psicothema2017.269

Mertens, V.-C., Bosma, H., Groffen, D. A. I. y van Eijk, J.T.M. (2011).

Good friends, high income or resilience? What matters

most for elderly patients? European Journal of Public Health, 22(5), 671-676. doi:10.1093/eurpub/ckr104

Nawaz, A., Malik, J. A. y Batool, A. (2014). Relationship between resilience and quality of life in diabetics. Journal of the College of Physicians and Surgeons Pakistan, 24(9), 670-675. doi:09.2014/JCPSP.670675

Organización Mundial de la Salud. (2016). Informe mundial sobre la diabetes. Recuperado de http://apps.who.int/iris/bitstream/ handle/10665/254649/9789243565255

Organización Mundial de la Salud. (2017). Enfermedades no transmisibles. Recuperado de http://www.who.int/mediacentre/factsheets/fs355/es/

Perna, L. et al. (2013). The association between resilience and diabetic neuropathy by socioeconomic position: Crosssectional findings from the KORA-Age study. Journal of Health Psychology, 20(9), 1222-1228. doi:10.1177/1359105313510334

Pervanidou, P. y Chrousos, G. P. (2012). Metabolic consequences of stress during childhood and adolescence. Metabolism, 61(5), 611-619. doi:10.1016/j.metabol.2011.10.005

Pervanidou, P., Agorastos, A., Kolaitis, G. y Chrousos, G. P. (2017). Neuroendocrine responses to early life stress and trauma and susceptibility to disease. European Journal of Psychotraumatology, 8(sup4). doi: $10.1080 / 20008198.2017 .1351218$

Pyatak, E. et al. (2016). Resilient, empowered, active living with diabetes (REAL Diabetes) study: Methodology and baseline characteristics of a randomized controlled trial evaluating an occupation-based diabetes management 
intervention for young adults. Contemporary Clinical Trials,

54, 8-17. doi:10.1016/j.cct.2016.12.025

Pyatak, E. et al. (2018). Occupational therapy intervention improves glycemic control and quality of life among young adults with diabetes: The resilient, empowered, active living with diabetes (REAL Diabetes) randomized controlled trial. Diabetes Care, 41(4), 696-704.

doi:10.2337/dc17-1634

Rendón, J. E. y Lugli, Z. (2013). Efectos del tipo de diabetes, estrategias de afrontameinto, sexo y optimismo en el apoyo social. Revista de Psicopatología y Psicología Clínica, 18(3), 193-207. doi:10.5944/rppc.vol.18.num.3.2013.12920

Richardson, G. (2002). The meta-theory of resilience and resiliency. Journal of Clinical Psychology, 58, 307-321. doi: $10.1002 /$ jclp. 10020

Speight, J., Browne, J. L., Holmes-Truscott, E., Hendrieckx, C. y Pouwer, F. (2012). Diabetes MILES-Australia (management and impact for long-term empowerment and success): methods and sample characteristics of a national survey of the psychological aspects of living with type 1 or type 2 diabetes in Australian adults. BMC Public Health, 12, 120. doi:10.1186/1471-2458-12-120

Steindhardt, M. A., Mamerow, M. M., Brown, S. A. y Jolly, C. A. (2009). A resilience intervention in African-American adults with type 2 diabetes. The Diabetes Educator, 35(2), 274-284. doi:10.1177/0145721708329698

Steinhardt, M. A., Brown, S.A., Dubois, S. K., Harrison, Jr., L., Lehrer, H. M. y Jaggars, S.S. (2015). A resilience intervention in African-American adults with type 2 diabetes. Behavior, 39(4), 507-518. doi:10.5993/AJHB.39.4.7 Steinhardt, M.A. et al. (2015). Positivity and indicators of health among African-Americans with diabetes. American Journal of Health Behavior, 39(1), 43-50. doi:10.5993/AJHB.39.1.5 
Steptoe, A. (2016). Stress resilience and risk of type 2 diabetes mellitus. Nature Reviews Endocrinology, 12(4), 189-190. doi:10.1038/nrendo.2016.17

Stuckey, H. L. et al. (2014). Personal accounts of the negative and adaptive psychosocial experiences of people with diabetes in the second diabetes attitudes, wishes and needs (DAWN2) study. Diabetes Care, 37, doi:10.2337/dc13$\underline{2536}$

Unantenne, N., Warren, N., Canaway, R. y Manderson, L. (2011). The strength to cope: Spirituality and faith in chronic disease. Journal of Religion \& Health, 52(4), 1147-1161. doi:10.1007/s10943-011-9554-9

Velthorst, E., Reichenberg, A., Rabinowitz, J. y Levine, S. (2015). Study of resilience and environmental adversity in midlife health (STREAM). Social Psychiatry and Psychiatric Epidemiology, 50(12), 1915-1922. doi:10.1007/s00127-0151126-y

Willrich J., Guerreiro, D. y Hegadoren, K. (2016).

Sociodemographic factors and health conditions associated with the resilience of people with chronic diseases: a cross sectional study. Revista Latino-Americana de Enfermagem, 24, doi:10.1590/1518-8345.1205.2786

Yi, J., Vitaliano, P., Smith, R., Yi, J. y Weinger, K. (2008). The role of resilience on psychological adjustment and physical health in patients with diabetes. British Journal of Health Psychology, 13(2), 311-325. doi:10.1348/135910707x186994

\section{ACERCA DEL AUTOR}

Ana María Sandoval Jaramillo (anasandy2004@yahoo.com.mx) es licenciada en Psicología Clínica por la Universidad de 
Guanajuato. Cursa el último semestre de la Maestría en Ciencias del Comportamiento, de la División Ciencias de la Salud en la Universidad de Guanajuato. Trabaja como psicóloga en la Clínica de Medicina Familiar, en León, del Instituto de Seguridad y Servicios Sociales para los Trabajadores del Estado. Es docente en el Departamento de Psicología de la División Ciencias de la Salud de la Universidad de Guanajuato (ORCID 0000-0003-3825-8595).

Recibido: 22/03/2019 Aceptado: 04/05/2019

CÓMO CITAR ESTE ARTÍCULO

Sandoval Jaramillo, A. M. (2019). La resiliencia en adultos con diabetes tipo 2: revisión teórica (2007-2018). Caleidoscopio Revista Semestral de Ciencias Sociales y Humanidades, 32(41), 105130. doi:10.33064/41crscsh1830 